08

\title{
Высокочастотная ЭПР-спектроскопия парамагнитных центров марганца в кристаллах GaAs: Mn
}

\author{
(ㄱ Р.А. Бабунц, А.С. Гурин, И.В. Ильин, А.П. Бундакова, М.В. Музафарова, \\ А.Г. Бадалян, Н.Г. Романов , П.Г. Баранов \\ Физико-технический институт им. А.Ф.Иоффре РАН, \\ Санкт-Петербург, Россия \\ `E-mail: nikolai.romanov@mail.ioffe.ru
}

Поступила в Редакцию 15 июня 2021 г.

В окончательной редакции 15 июня 2021 г.

Принята к публикации 16 июня 2021 г.

\begin{abstract}
Методы высокочастотного электронного парамагнитного резонанса (ЭПР) использованы для исследования уникальных свойств центров марганца в кристалле GaAs:Mn в сильных магнитных полях при низких температурах. На частотах 94 и $130 \mathrm{GHz}$ зарегистрированы переходы ЭПР в комплексе, представляющем собой ион марганца, замещающий $\mathrm{Ga}$, и являющийся ионизированным акцептором $\mathrm{Mn}_{\mathrm{Ga}}^{2+}\left(A^{-}\right)$, который связан изотропным обменным взаимодействием с делокализованной (мелкой) дыркой $\left(\mathrm{Mn}_{\mathrm{Ga}}^{2+} \mathrm{SH}\right.$ (shallow hole)). В таком комплексе $\mathrm{Mn}_{\mathrm{Ga}}^{2+}$ со спином $S=5 / 2$ связан антиферромагнитным обменным взаимодействием с мелкой дыркой с угловым моментом $J=3 / 2$. В результате сложения угловых моментов $\mathbf{F}=\mathbf{S}+\mathbf{J}$ имеются четыре энергетических уровня с $F=1,2,3,4$, характеризующихся вырождение $2 F+1$ в нулевом магнитном поле, и с нижним уровнем $F=1$. Проанализирована сложная система энергетических уровней этого комплекса в магнитном поле и возможность точного определения обменных взаимодействий по спектрам ЭПР. Исследован другой комплекс на основе ионизированного акцептора $\mathrm{Mn}_{\mathrm{Ga}}^{2+}$, взаимодействующего с локализованным дырочным центром, в виде диамагнитного иона $\mathrm{O}^{2-}$, замещающего Аs. Этот комплекс, $\mathrm{Mn}_{\mathrm{Ga}}^{2+}-\mathrm{O}_{\mathrm{As}}^{2-}$, характеризуется аксиальной симметрией вдоль оси $\langle 111\rangle$ кубического кристалла $\mathrm{GaAs} \mathrm{c}$ анизотропным спектром ЭПР. Благодаря высокому фактору Больцмана, в наших исследованиях был определен порядок спиновых уровней тонкой структуры этого комплекса. Влияние больцмановского распределения населенностей уровней продемонстрировано также для комплекса $\mathrm{Mn}_{\mathrm{Ga}}^{2+} \mathrm{SH}$.
\end{abstract}

Ключевые слова: высокочастотный ЭПР, кристалл $\mathrm{GaAs}$, акцептор $\mathrm{Mn}$, мелкая дырка, обменное взаимодействие.

DOI: 10.21883/FTT.2021.11.51596.146

\section{1. Введение}

Практически все применения полупроводниковых материалов требуют их легирования электрически активными примесями, создающими электронную $(n)$ и дырочную $(p)$ проводимость, либо обусловливающими их полуизолирующие свойства. Развитие новой области спинтроники, требует внедрения магнитных материалов в приборные структуры, однако такие применения ограничены совместимостью магнитных материалов с основными полупроводниками, например, с GaAs. Естественным направлением развития этой области является легирование полупроводниковых материалов магнитными примесями, так называемые „полумагнитные“ полупроводники, в которых магнитные свойства проявляются непосредственно внутри полупроводника. При их использовании в электронных и оптических полупроводниковых устройствах добавляется новая степень свободы, связанная со спином носителей. Магнитные примеси в виде ионов переходных элементов могут быть введены в полупроводники типа II-VI в высоких концентрациях, поэтому основное внимание по созданию полумагнитных полупроводников было обращено на эти соединения [1-3]. Однако к настоящему времени наиболее перспективной считается система GaAs: Mn, которая продемонстрировала ферромагнитные свойства при достаточно высокой температуре, оставаясь при этом полупроводниковым материалом $p$-типа $[4,5]$. Прямозонный полупроводник $\mathrm{GaAs} \mathrm{c}$ шириной запрещенной $1.43 \mathrm{eV}$ является основным материалом в оптоэлектронике для использования в оптической связи, при этом, как электронные устройства, так и оптические излучатели работают при комнатной температуре. $\mathrm{B}$ GaAs: Mn реализуются несколько типов парамагнитных центров марганца, при этом центральное место занимает необычная система в виде иона $\mathrm{Mn}^{2+}$, замещающего $\mathrm{Ga}$, и являющегося акцептором $\mathrm{Mn}_{\mathrm{Ga}}^{2+}\left(A^{-}\right)$, который связан изотропным обменным взаимодействием с делокализованной дыркой с мелкими уровнями [6-9]. В результате создается мелкий акцептор, в котором магнитный ион непосредственно связан с электрически активным акцептором (см. обзор [9]).

Разбавленный магнитный полупроводник $(\mathrm{Ga}, \mathrm{Mn}) \mathrm{As}$ [5] совместим с обычным полупроводником $\mathrm{GaAs}$, что позволило рассматривать его в качестве идеального материала для исследований и возможных 
применений в спинтронике. Таким образом, легирующая примесь $\mathrm{Mn}$ в $\mathrm{GaAs}$ является мелким акцептором, одновременно создающим магнитный ион и дырку. Ферромагнетизм в GaAs:Mn возникает при введении высокой (более 1\%) концентрации магнитных ионов $\mathrm{Mn}^{2+}$. Считается, что ферромагнитная связь между спинами $S=5 / 2$ ионов $\mathrm{Mn}^{2+}$ возникает благодаря наличию делокализованных дырок, являющихся своеобразным „клеем“ [10].

Задачей настоящей работы является проведение исследований центров марганца в высоких магнитных полях и низких температурах, т.е. в условиях, когда заселены только нижние спиновые уровни в соответствии с распределением Больцмана.

\section{2. Методика эксперимента}

Объемный кристалл GaAs:Mn был выращен методом Чохральского и легирован $\mathrm{Mn}$ с концентрацией $\sim 10^{18} \mathrm{~cm}^{-3}$. Заготовка в форме шайбы выращивалась в направлении [111]. Образцы вырезались в виде параллелепипеда с размерами $\sim 3 \times 3 \times 1 \mathrm{~mm}$ и устанавливались таким образом, чтобы можно было вращать их вокруг ребра, совпадающего с одним из направлений $\langle 110\rangle$ кристалла.

Исследования ЭПР проводились в трех диапазонах: стандартном $3 \mathrm{~cm}$ диапазоне (частота $9.4 \mathrm{GHz}$ ) и высокочастотных диапазонах $3 \mathrm{~mm}(94 \mathrm{GHz})$ и $2 \mathrm{~mm}(130 \mathrm{GHz})$. Для регистрации спектров ЭПР в высокочастотных $3 \mathrm{~mm}$ и $2 \mathrm{~mm}$ диапазонах, был применен спектрометр, созданный в ФТИ им. А.Ф. Иоффе в кооперации с компанией ДОК. Спектрометр работает в непрерывном и импульсном режимах, диапазон изменения магнитных полей 0-7 Т, диапазон изменения температуры $1.5-300 \mathrm{~K}$. Наряду со стандартной методикой регистрации спектров ЭПР с низкочастотной модуляцией магнитного поля, был добавлен режим с низкочастотной модуляцией частоты, который дает преимущества при работе в сильных магнитных полях, так как исключаются шумы, связанные с воздействием сильных магнитных полей на модуляционные катушки.

\section{3. Экспериментальные результаты и обсуждение результатов}

На рис. 1 представлены спектры ЭПР центров марганца в кристалле GaAs: Mn, зарегистрированные при температурах $1.7-4.2 \mathrm{~K}$ в трех диапазонах: в высокочастотных $2 \mathrm{~mm}$ диапазоне $130 \mathrm{GHz}(a)$ и $3 \mathrm{~mm}$ диапазоне $94 \mathrm{GHz}(b)$, а также в стандартном $3 \mathrm{~cm}$ диапазоне $9.4 \mathrm{GHz}(c)$. Зарегистрированы три типа центров марганца: (i) ионы $\mathrm{Mn}^{2+}$, занимающие позиции $\mathrm{Ga}, \mathrm{Mn}_{\mathrm{Ga}}^{2+}$, являющиеся отрицательно заряженными акцепторами $A^{-}$, захватившие мелкие дырки, делокализованные вокруг $\mathrm{Mn}_{\mathrm{Ga}}^{2+}$, как кулоновского центра притяжения; (описанный выше комплекс $\mathrm{Mn}_{\mathrm{Ga}}^{2+}-\mathrm{SH}$ ); (ii) ионы $\mathrm{Mn}_{\mathrm{Ga}}^{2+}$, являющиеся отрицательно заряженными акцепторами $A^{-}\left(\mathrm{Mn}_{\mathrm{Ga}}^{2+}\right)$; (iii) ион $\mathrm{Mn}^{2+}$, занимающий позицию $\mathrm{Ga}, \mathrm{Mn}_{\mathrm{Ga}}^{2+}$ и являющийся отрицательно заряженным акцептором $A^{-}$, в ближайшем окружении которого вдоль молекулярной связи тетраэдра в направлении $\langle 111\rangle$ располагается кислород в непарамагнитном состоянии $\mathrm{O}^{2-}$, замещающий As- $\mathrm{O}_{\mathrm{As}}^{2-}$, (комплекс $\mathrm{Mn}_{\mathrm{Ga}}^{2+}-\mathrm{O}_{\mathrm{As}}^{2-}$ ). Модели трех рассмотренных центров марганца представлены на рис. 2, где условно показан примерный радиус делокализованной дырки с центром на ионе $\mathrm{Mn}_{\mathrm{Ga}}^{2+}$, который, как показано в работе [9], имеет величину порядка $1 \mathrm{~nm}$. Таким образом, мелкая дырка распространяется на несколько элементарных ячеек кубического кристалла GaAs. Представляет особый интерес исследования комплексов $\mathrm{Mn}_{\mathrm{Ga}}^{2+}-\mathrm{SH}$ в наноструктурах, где ожидается проявление эффектов конфайнмента, которые неизбежно приведут к изменениям в обменном взаимодействии между спиновой системой марганца и угловым моментом мелкой дырки.

На рис. 1,c также приведены спектры ЭПР, зарегистрированные в $3 \mathrm{~cm}$ диапазоне в дополнительном образце, в котором наблюдается разрешенная сверхтонкая (CT) структура, как для ионов $\mathrm{Mn}_{\mathrm{Ga}}^{2+}$ в бездефектном окружении, так и для комплексов $\mathrm{Mn}_{\mathrm{Ga}}^{2+}-\mathrm{O}_{\mathrm{As}}^{2-}$. При этом видно, что величина СТ расщепления в обоих центрах примерно одинаковая. В этом образце наблюдается только запрещенный переход для комплекса $\mathrm{Mn}_{\mathrm{Ga}}^{2+}-\mathrm{SH}$, разрешенный переход не виден, по-видимому, из-за сильных напряжений в кристалле. На этом рисунке также для сравнения пунктирной линией показан спектр, подобный, приведенному в работе [6], в которой спектры ЭПР комплексов $\mathrm{Mn}_{\mathrm{Ga}}^{2+}$-SH были зарегистрированы в стандартном $3 \mathrm{~cm}$ диапазоне. Эти измерения не позволили непосредственно обнаружить влияние на спектр ЭПР следующего, возбужденного состояния комплекса $\mathrm{Mn}_{\mathrm{Ga}}^{2+}-\mathrm{SH}$ с $F=2$, на его существование указывали только косвенные данные.

В настоящей работе измерения проводятся в высоких магнитных полях и при низких температурах, т.е. в условиях, когда заселены только нижние спиновые уровни. При этом появляется возможность тестирования сложной системы энергетических уровней комплекса $\mathrm{Mn}_{\mathrm{Ga}}^{2+}-\mathrm{SH}$, а также системы уровней комплекса $\mathrm{Mn}_{\mathrm{Ga}}^{2+}-\mathrm{O}_{\mathrm{As}}^{2-}$. В обоих случаях комплексы представляют собой центр марганца, $\mathrm{Mn}_{\mathrm{Ga}}^{2+}$, являющийся отрицательно заряженным (ионизированным) акцептором $A^{-}$, который притягивает или делокализованную мелкую дырку (shallow hole) или дырку, локализованную на соседнем атоме в виде примесного иона кислорода в диамагнитном состоянии $\mathrm{O}_{\mathrm{As}}^{2-}$. Первый комплекс приводит к появлению изотропного спектра ЭПР, во втором случае спектр ЭПР становится анизотропным.

Рассмотрим комплекс $\mathrm{Mn}_{\mathrm{Ga}}^{2+} \mathrm{SH}$. Его общепринятой моделью является система, в которой дырке энергетически выгодно оставаться на делокализованной орбите вокруг акцептора $A^{-}$в виде $\mathrm{Mn}_{\mathrm{Ga}}^{2+}\left(3 d^{5}\right), S=5 / 2$ (рис. 2, 

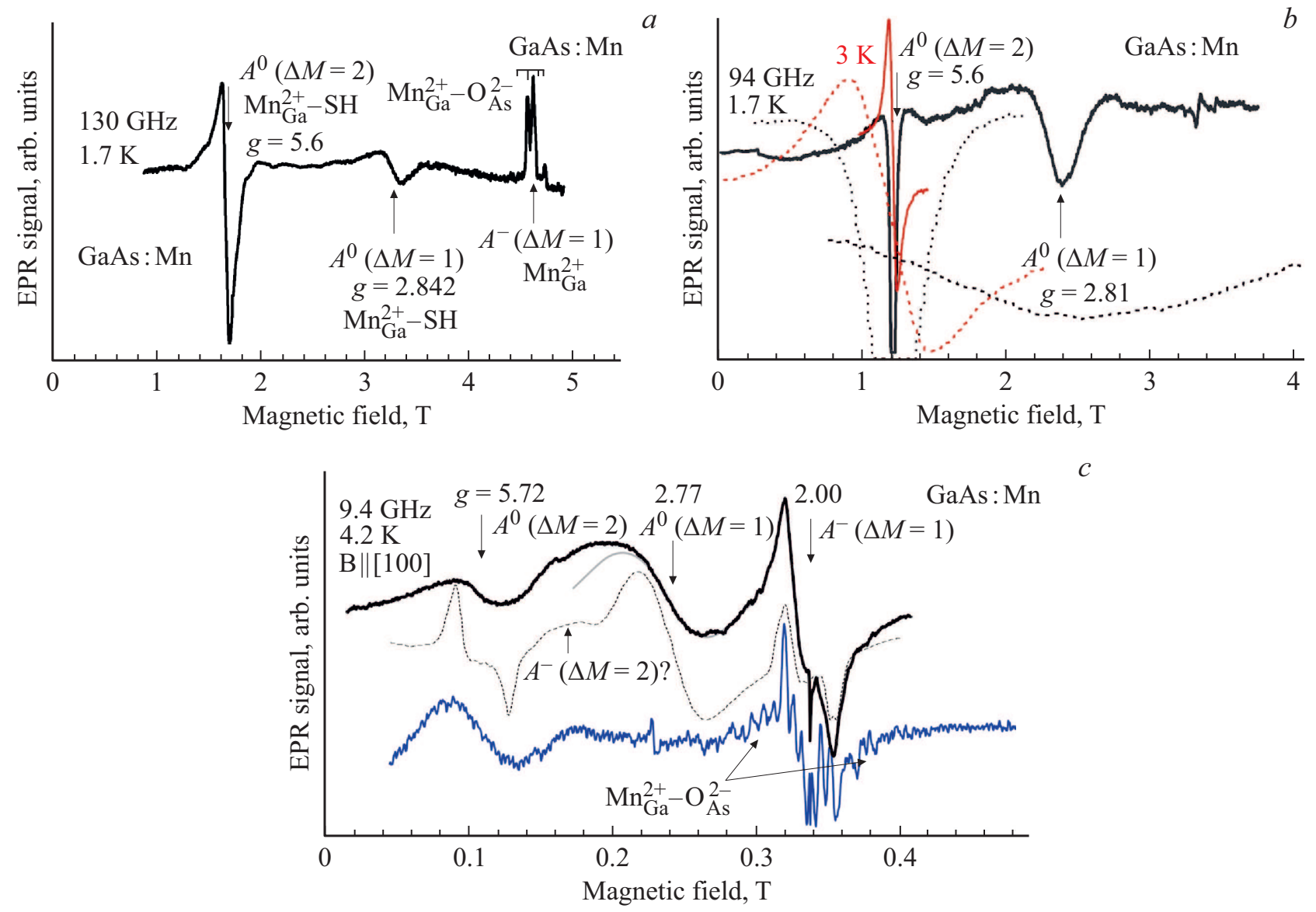

Рис. 1. Спектры ЭПР-центров марганца в кристалле GaAs:Mn, зарегистрированные в трех диапазонах при температуре 1.7-4.2 K: (a) $2 \mathrm{~mm}$ диапазоне $130 \mathrm{GHz}$; (b) $3 \mathrm{~mm}$ диапазоне $94 \mathrm{GHz}$ (красной линией отдельно показан запрещенный переход, зарегистрированный при $3 \mathrm{~K})$ и $(c) 3 \mathrm{~cm}$ диапазоне $9.4 \mathrm{GHz}$. Зарегистрированы три типа центров марганца: (i) ион Мn ${ }^{2+}$, занимающий позицию $\mathrm{Ga}, \mathrm{Mn}_{\mathrm{Ga}}^{2+}$, и являющийся отрицательно заряженным акцептором $A^{-}$, захватившим мелкую дырку, делокализованную вокруг $\mathrm{Mn}_{\mathrm{Ga}}^{2+}$, как кулоновского центра притяжения; $\mathrm{Mn}_{\mathrm{Ga}}^{2+} \mathrm{SH}$; (ii) ион $\mathrm{Mn}_{\mathrm{Ga}}^{2+}$, являющийся отрицательно заряженным акцептором $A^{-}$; (iii) ион $\mathrm{Mn}_{\mathrm{Ga}}^{2+}$ в ближайшем окружении которого вдоль молекулярной связи тетраэдра в направлении $\langle 111\rangle$ располагается кислород в непарамагнитном состоянии $\mathrm{O}^{2-}$, замещающий $\mathrm{As}, \mathrm{O}_{\mathrm{As}}^{2-}$, комплекс $\mathrm{Mn}_{\mathrm{Ga}}^{2+}-\mathrm{O}_{\mathrm{As}}^{2-}$. На $(c)$ приведены спектры ЭПР, зарегистрированные в $3 \mathrm{~cm}$ диапазоне в том же образце, а также в дополнительном образце, в котором наблюдается разрешенная сверхтонкая структура, как для ионов $\mathrm{Mn}_{\mathrm{Ga}}^{2+}$ в бездефектном окружении, так и для комплексов $\mathrm{Mn}_{\mathrm{Ga}}^{2+}-\mathrm{O}_{\mathrm{As}}^{2-}$. Штриховой линией показан спектр подобный приведенному в работе [6], в которой спектры ЭПР для комплексов $\mathrm{Mn}_{\mathrm{Ga}}^{2+}-\mathrm{SH}$ были зарегистрированы в стандартном $3 \mathrm{~cm}$ диапазоне. Для сравнения на $(b)$ штриховыми линиями показаны спектры ЭПР для комплексов $\mathrm{Mn}_{\mathrm{Ga}}^{2+} \mathrm{SH}$ на частоте $94 \mathrm{GHz}$ (отдельно разрешенные и запрещенные переходы), представленные в том же масштабе, в котором приведены спектры на $(c)$ для $3 \mathrm{~cm}$ диапазона, то есть в десять раз меньшем.

левая часть). В этом случае комплекс $\mathrm{Mn}_{\mathrm{Ga}}^{2+}$ плюс мелкая дырка образуется в результате изотропного обменного взаимодействия между электронами $3 d^{5}$-оболочки иона $\mathrm{Mn}_{\mathrm{Ga}}^{2+}(S=5 / 2)$ и спинами делокализованной дырки $(J=3 / 2)$. В результате образуется мелкий акцептор с глубиной уровня порядка $0.1 \mathrm{eV}$ [6-9].

Спектр ЭПР комплекса $\mathrm{Mn}_{\mathrm{Ga}}^{2+}-\mathrm{SH}$ в $\mathrm{GaAs}$ может быть описан приближенным спиновым гамильтонианом

$$
H=g_{S} \mu_{\mathrm{B}} \mathbf{B} \cdot \mathbf{S}+g_{J} \mu_{\mathrm{B}} \mathbf{B} \cdot \mathbf{J}+c \mathbf{S} \cdot \mathbf{J}
$$

где $B$ - магнитное поле, $g_{S}-$ изотропный $g$-фактор акцептора $\mathrm{Mn}_{\mathrm{Ga}}^{2+}\left(3 d^{5}, S=5 / 2\right)$ равный $2.00, \mu_{\mathrm{B}}-$ магнетон Бора, $g_{J}-g$-фактор мелкой дырки $g_{J}=0.78$ [11], $c$ - изотропное обменное взаимодействие между $\mathrm{Mn}_{\mathrm{Ga}}^{2+}$ и мелкой дыркой.

В рассматриваемом комплексе $\mathrm{Mn}_{\mathrm{Ga}}^{2+}$ со спином $S=5 / 2$ связан антиферромагнитным обменным взаимодействием с (в виде $c \mathbf{S} \cdot \mathbf{J})$ с мелкой дыркой с угловым моментом $J=3 / 2$. В результате по правилу сложения моментов $\mathbf{F}=\mathbf{S}+\mathbf{J}(F=1,2,3,4)$, где в соответствие с правилом интервалов Ландэ $\Delta E_{F-(F-1)}=c F$, то есть, интервалы между уровнями в нулевом магнитном поле равны соответственно $2 c, 5 c(2 c+3 c)$, $9 c(2 c+3 c+4 c)$, начиная с нижнего уровня. Таким образом, основное состояние $F=1$ получается, если предположить антиферромагнитную связь дырки с акцепторным ядром $3 d^{5}$. Соответствующие собственные 

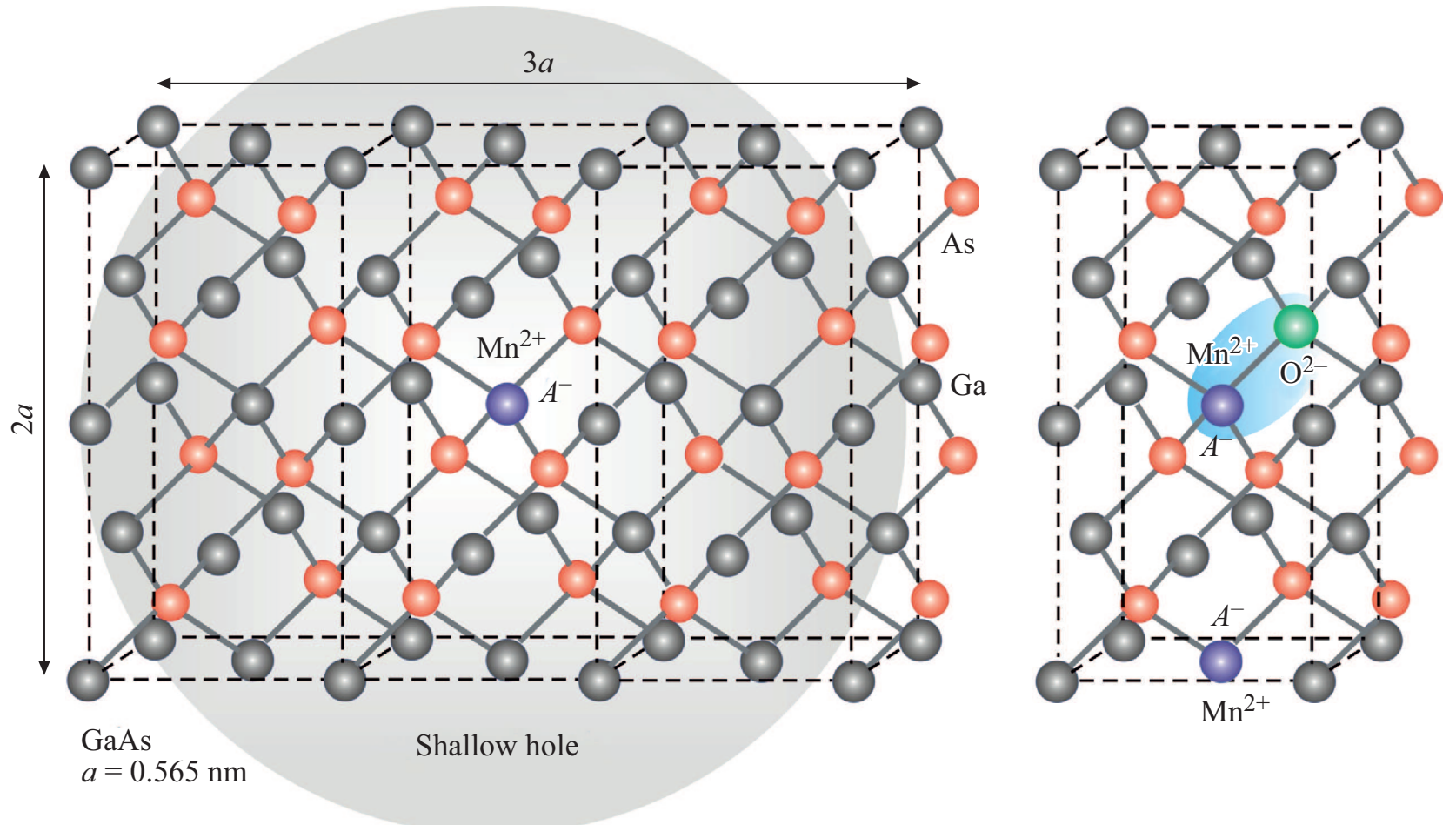

Рис. 2. Модели трех центров марганца в кристалле $\mathrm{GaAs}: \mathrm{Mn}$ : (левая часть) комплекс $\mathrm{Mn}_{\mathrm{Ga}}^{2+}-\mathrm{SH}$; (правая часть) $\mathrm{Mn}_{\mathrm{Ga}}^{2+}$ в бездефектном окружении и комплекс $\mathrm{Mn}_{\mathrm{Ga}}^{2+}-\mathrm{O}_{\mathrm{As}}^{2-}$. Серым цветом для комплекса $\mathrm{Mn}_{\mathrm{Ga}}^{2+}-\mathrm{SH}$ условно выделено пространственное распространение делокализованной мелкой дырки внутри кристалла.

векторы состояния $F=1$ в базисе $\left|M_{S}, M_{J}\right\rangle$ вычисляются как $[6,9]$.

$$
\begin{aligned}
|1,+1\rangle & =1 / 2 \sqrt{ } 2|5 / 2,-3 / 2\rangle-1 / 10 \sqrt{ } 30|3 / 2,-1 / 2\rangle \\
& +1 / 10 \sqrt{ } 15|1 / 2,1 / 2\rangle-1 / 10 \sqrt{ } 5|-1 / 2,3 / 2\rangle \\
|1,0\rangle & =1 / 5 \sqrt{ } 5|3 / 2,-3 / 2\rangle-1 / 10 \sqrt{ } 30|1 / 2,-1 / 2\rangle \\
& +1 / 10 \sqrt{ } 30|-1 / 2,1 / 2\rangle-1 / 5 \sqrt{ } 5|-3 / 2,3 / 2\rangle, \\
|1,-1\rangle & =-1 / 2 \sqrt{ } 2|-5 / 2,3 / 2\rangle+1 / 10 \sqrt{ } 30|-3 / 2,1 / 2\rangle \\
& -1 / 10 \sqrt{ } 15|-1 / 2,-1 / 2\rangle+1 / 10 \sqrt{ } 5|1 / 2,-3 / 2\rangle
\end{aligned}
$$

В результате расчетов с использованием волновых функций нижнего триплетного уровня с $F=1$ (2), получаем изотропный $g$-фактор:

$$
g_{F=1} \approx(7 / 4) g_{S}-(3 / 4) g_{J}
$$

С учетом приведенных выше значений $g_{S}$ и $g_{J}$ получаем из выражения (3) $g_{F=1}=2.9$. Это значение соответствует величине $g_{F=1}=2.77$, измеренной по спектрам ЭПР [6] и $g=2.74$, определенной с помощью комбинационного рассеяния света с переворотом спина (SFRS) [12], что подтверждает используемую модель в виде $\mathrm{Mn}_{\mathrm{Ga}}^{2+}-\mathrm{SH}$. В спектрах ЭПР [6] наблюдались две линии; соответствующие переходам $\Delta M_{F}=1$ и $\Delta M_{F}=2$ между подуровнями основного состояния $F=1$ с $g$-факторами $g=2.77$ и $g=5.72$ соответственно.

Основное состояние $F=1$ разбивается на два мультиплета с проекциями полного углового момента на ось квантования $M_{F}=0$ и $M_{F}= \pm 1$ из-за случайного локального напряжения. Это расщепление меньше обменной энергии акцептора $\mathrm{Mn}$, которая принимает значения в диапазоне 4-6 meV как было оценено по данным различных косвенных исследований $[13,14]$. Прямые измерения разности энергий между состояниями $F=1$ и $F=2$ методом SFRS дали оценку обменной константы $\Delta\left(F_{1}-F_{2}\right)=2.2 \mathrm{meV}\left(17 \mathrm{~cm}^{-1}\right)[12]$.

На рис. 1, $b$ штрихами показаны раздельно разрешенные и запрещенные переходы в спектрах ЭПР комплексов $\mathrm{Mn}_{\mathrm{Ga}}^{2+}$-SH на частоте $94 \mathrm{GHz}$, представленные для сравнения в том же масштабе, в котором приведены спектры на рис. 1, с для $3 \mathrm{~cm}$ диапазона, т.е. в десять раз меньшем. Видно, что ширины линий запрещенного перехода почти не отличаются, при этом ширина линии разрешенного перехода в $3 \mathrm{~mm}$ диапазоне существенно больше по сравнению с $3 \mathrm{~cm}$ диапазоном. Малое изменение ширины линии запрещенного перехода при увеличении магнитных полей на порядок свидетельствует о том, что в комплексе $\mathrm{Mn}_{\mathrm{Ga}}^{2+}-\mathrm{SH}$, имеется малый разброс $g$-факторов. Разрешенный переход обычно сильно уширен из-за напряжений в кристалле, которые приводят к расщеплению тонкой структуры в состоянии мелкой 


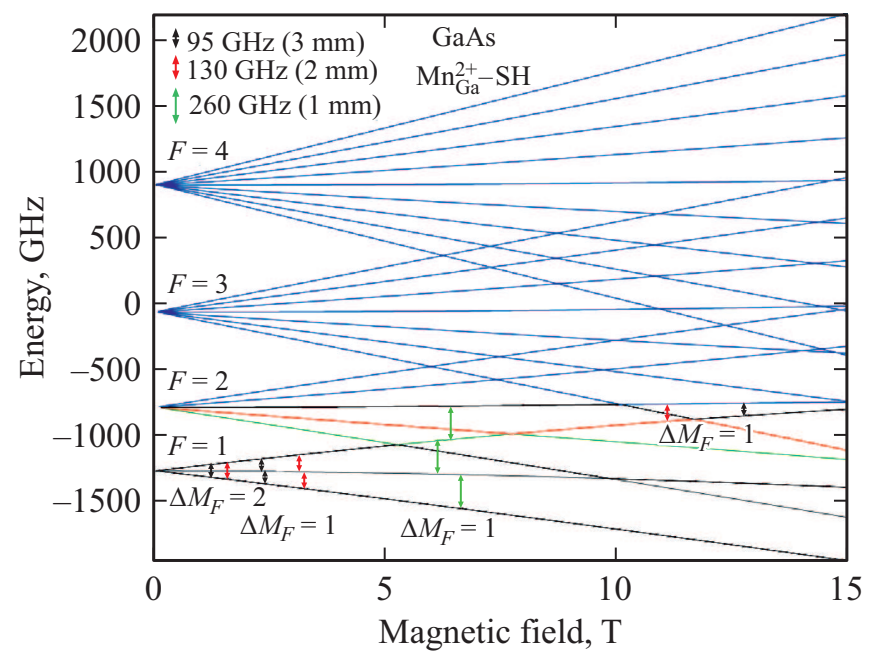

Рис. 3. Схема энергетических уровней для центра $\mathrm{Mn}_{\mathrm{Ga}}^{2+}-\mathrm{SH}$, представляющего собой ион $\mathrm{Mn}_{\mathrm{Ga}}^{2+}$ со спином $S=5 / 2$, связанный антиферромагнитным обменным взаимодействием $c$ с мелкой дыркой с угловым моментом $J=3 / 2$. Каждый из четырех уровней $F=1,2,3,4$ в нулевом магнитном поле (без учета расщеплений, вызванных напряжениями в кристалле) характеризуются $2 F+1$ кратным вырождением, которое снимается магнитным полем. Для диапазона $130 \mathrm{GHz}$ при низкой температуре видны три перехода в доступных магнитных полях, два разрешенных в поле порядка $3 \mathrm{~T}$ и один запрещенный в половинном поле. В полях порядка 12 Т виден еще один разрешенный переход между уровнем $M_{F=1}=+1$ и $M_{F=2}=0$, но этот переход находится за пределами возможностей ЭПР-спектрометра. Показаны также переходы для диапазона $94 \mathrm{GHz}$. Также приведены результаты расчета для ожидаемых переходов в 1 мм диапазоне на частоте $260 \mathrm{GHz}$.

дырки, вызывающей также расщепление уровней с $F=1$ в нулевом магнитном поле.

На рис. 3 приведена схема энергетических уровней для центра $\mathrm{Mn}_{\mathrm{Ga}}^{2+}-\mathrm{SH}$, представляющего собой $\mathrm{Mn}_{\mathrm{Ga}}^{2+}$ со спином $S=5 / 2$, связанный антиферромагнитным обменным взаимодействием $c$ с мелкой дыркой с угловым моментом $J=3 / 2$. Все четыре уровня в нулевом магнитном поле (без учета расщеплений, вызванных напряжениями в кристалле) характеризуются $2 F+1$ кратным вырождением, которое снимается магнитным полем. В магнитном поле нижний уровень с $F=1$, являющийся триплетом, расщепляется на подуровни с $M_{F}=+1,0,-1$. Мы будем рассматривать только нижний триплет, так как верхние уровни не заселены в условиях настоящих экспериментов. Для диапазона $130 \mathrm{GHz}$ видны три перехода в доступных магнитных полях, два разрешенных в поле порядка 3 Т и один запрещенный в половинном поле. Эти переходы и наблюдаются в эксперименте для комплекса $\mathrm{Mn}_{\mathrm{Ga}}^{2+} \mathrm{SH}$. Видно, что разрешенные переходы расщеплены, что обусловлено нелинейностью уровня $M_{F=1}=0$ в магнитном поле, который отклоняется от горизонтального направления вниз на энергетической шкале, расходясь по энергиям с уровнем $M_{F=2}=0$, который отклоняется вверх по энергиям. Величина расщепления разрешенных переходов непосредственно связана с влиянием верхнего уровня с $F=2$, то есть определяется величиной обменного расщепления. Из-за большой ширины линии разрешенных переходов (рис. 1,a,b), вызванной напряжениями в кристалле, это расщепление не разрешается в наших экспериментах даже на частоте $130 \mathrm{GHz}$. В полях порядка 12 Т виден еще один разрешенный переход на частоте $130 \mathrm{GHz}$ между уровнем $M_{F=1}=+1$ и $M_{F=2}=0$, но этот переход находится за пределами возможностей ЭПРспектрометра. На частоте $94 \mathrm{GHz}$ этот переход должен наблюдаться в еще более высоких полях. Однако, если увеличить частоту до $260 \mathrm{GHz}$ (1 mm диапазон), то этот разрешенный переход попадает в область доступных магнитных полей, как видно из рис. 3. В этом диапазоне можно будет с большой точностью определить величину обменного взаимодействия в комплексе $\mathrm{Mn}_{\mathrm{Ga}}^{2+} \mathrm{SH}$ по спектрам ЭПР.

При расчетах запрещенные переходы $\Delta M_{F}=2$, показанные на рис. 3, появляются только, если задавать напряжения в кристалле, приводящие к расщеплению уровней $F=1$ в нулевом магнитном поле, однако, интенсивность этих переходов является чрезвычайно низкой. Тем не менее мы видим, что и при высоких магнитных полях, рис. $1, a, b$, запрещенные переходы чрезвычайно эффективны. Последнее поддерживает предположение, высказанное в работе [15], что запрещенные переходы вызываются электрической компонентой микроволнового поля. В наших экспериментах длина волны 2 и $3 \mathrm{~mm}$ сравнима с размерами образца, поэтому в возбуждении переходов между уровнями могут участвовать обе компоненты микроволнового поля. В работе [15] предложен новый механизм переходов ЭПР в системе $\mathrm{Mn}_{\mathrm{Ga}}^{2+}-\mathrm{SH}$, а именно, электро-дипольный, поскольку магнитно-дипольным переходам в спектрах ЭПР должны соответствовать линии, на несколько порядков менее интенсивные, по сравнению с наблюдаемыми в экспериментальных спектрах.

В самом деле, мы считаем, что этот механизм может быть предпочтительным, поскольку при моделировании спектров ЭПР получали очень низкую интенсивность запрещенного перехода $\Delta M_{F}=2$, при этом, чтобы его удалось наблюдать, необходимо было добавить в систему с $J=3 / 2$ расщепление тонкой структуры, которая, очевидно, имеет место из-за напряжений в кристалле.

На рис. 4, $a$ показан запрещенный переход ЭПРцентров $\mathrm{Mn}_{\mathrm{Ga}}^{2+} \mathrm{SH}$ в кристалле $\mathrm{GaAs}: \mathrm{Mn}$, зарегистрированный в $3 \mathrm{~mm}$ диапазоне $(95 \mathrm{GHz})$ при разных температурах. Видно, что при повышении температуры интенсивность сигнала ЭПР резко уменьшается. Температурная зависимость интенсивности сигнала ЭПРцентра $\mathrm{Mn}_{\mathrm{Ga}}^{2+}-\mathrm{SH}$ показана на рис. 4, $b$. Отметим, что температура исчезновения спектра ЭПР рассматриваемых комплексов, ранее наблюдаемая в стандартном $3 \mathrm{~cm}$ диапазоне [6], была порядка 10-12 K, т. е., как следует из наших исследований, не зависит от диапазона 

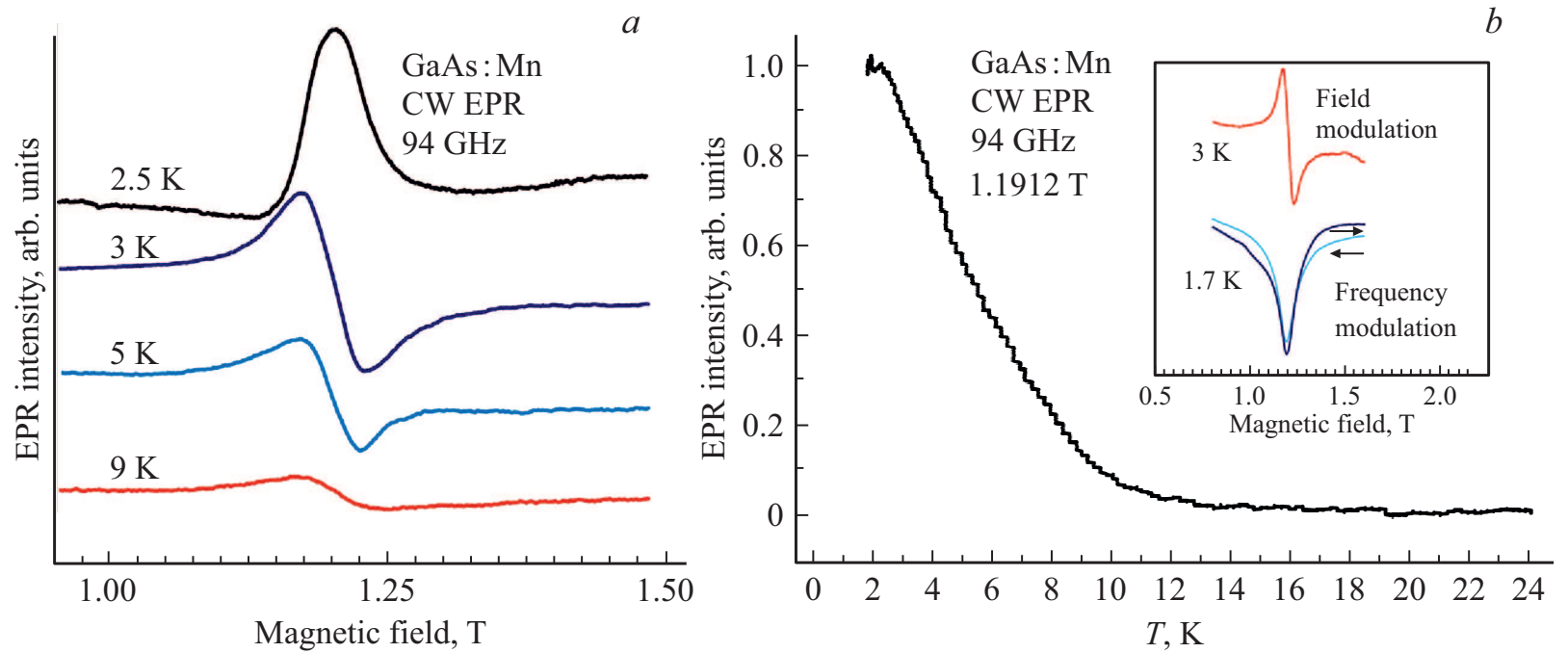

Рис. 4. (а) Запрещенный переход ЭПР-центров $\mathrm{Mn}_{\mathrm{Ga}}^{-} \mathrm{SH}$ в кристалле $\mathrm{GaAs}: \mathrm{Mn}$, зарегистрированный в $3 \mathrm{~mm}$ диапазоне (94 GHz) при разных температурах. (b) Температурная зависимость интенсивности сигнала ЭПР-центра $\mathrm{Mn}_{\mathrm{Ga}}^{2+}-\mathrm{SH}$. На вставке приведены сигналы ЭПР, зарегистрированные с низкочастотной модуляцией магнитного поля и низкочастотной частотной модуляцией, в обоих случаях использовалась частота модуляции $680 \mathrm{~Hz}$. Спектры зарегистрированы при противоположных направлениях развертки внешнего магнитного поля, показанных стрелками. Так как в системе GaAs: Mn возможно проявление ферромагнитных свойств, отсутствие гистерезиса свидетельствует о том, что в данном случае магнитное упорядочение не происходит.

регистрации спектров ЭПР, т.е. от величины магнитного поля. Можно предположить, что эта температура обусловлена мелким уровнем акцептора и его ионизацией при повышении температуры. На вставке рис. $4, b$ приведены сигналы ЭПР, зарегистрированные с низкочастотной модуляцией магнитного поля и низкочастотной частотной модуляцией, в обоих случаях использовалась частота модуляции $680 \mathrm{~Hz}$. Спектры зарегистрированы при противоположных направлениях развертки внешнего магнитного поля, показанных стрелками. Так как в системе GaAs:Mn возможно проявление ферромагнитных свойств, отсутствие гистерезиса свидетельствует о том, что в данном случае магнитное упорядочение не происходит. По-видимому, концентрация марганца в исследуемом образце сравнительно мала для таких эффектов.

Спектр ЭПР самого простого дефекта - иона $\mathrm{Mn}^{2+}$, занимающий позицию $\mathrm{Ga}, \mathrm{Mn}_{\mathrm{Ga}}^{2+}$, и являющийся отрицательно заряженным (ионизированным) акцептором $A^{-}$, который ранее был изучен методом ЭПР в работах $[16,17]$, описывается спиновым гамильтонианом со спином $S=5 / 2$ в виде

$$
\begin{aligned}
H & =g \mu_{\mathrm{B}} \mathbf{B} \cdot \mathbf{S}+(1 / 6) a \\
& \times\left[S_{x}^{4}+S_{y}^{4}+S_{z}^{4}-1 / 5 S(S+1)\left(3 S^{2}+3 S-1\right)\right]+A \mathbf{I} \cdot \mathbf{S} .
\end{aligned}
$$

Первое слагаемое описывает зеемановское взаимодействие, второе слагаемое описывает взаимодействие электронного спина с кубическим кристаллическим полем, последнее слагаемое отражает сверхтонкое взаимодействие между магнитным моментом электрона и магнитным моментом ядра марганца $\left({ }^{55} \mathrm{Mn}\right.$, природное содержание $100 \%, I=5 / 2)$. Параметры спинового гамильтониана равны: $|A|=52.4 \cdot 10^{-4} \mathrm{~cm}^{-1}$, $|a|=14 \cdot 10^{-4} \mathrm{~cm}^{-1}[16,17]$. Эти сигналы ЭПР наблюдаются на рис. $1, a, b, c$, при этом на рис. $1, c$ видна разрешенная сверхтонкая структура. Модель этого центра в виде изолированного иона $\mathrm{Mn}_{\mathrm{Ga}}^{2+}$ показана на рис. 2 , правая сторона.

На рис. $1, a, b, c$ виден спектр ЭПР комплекса на основе акцептора $\mathrm{Mn}_{\mathrm{Ga}}^{2+}$, взаимодействующего с локализованным дырочным центром, в виде диамагнитного иона $\mathrm{O}_{\mathrm{As}}^{2-}$, замещающего As. Этот комплекс, $\mathrm{Mn}_{\mathrm{Ga}}^{2+}-\mathrm{O}_{\mathrm{As}}^{2-}$, спектр ЭПР которого ранее наблюдался в работах $[18,19]$, характеризуется аксиальной симметрией вдоль оси $\langle 111\rangle$ кубического кристалла GaAs c анизотропным спектром ЭПР и спином $S=5 / 2$. На рис. $5, a$ показан спектр ЭПР комплекса марганца $\mathrm{Mn}_{\mathrm{Ga}}^{2+} \mathrm{O}_{\mathrm{As}}^{2-}$ в кристалле GaAs: Mn, зарегистрированный в $2 \mathrm{~mm}$ диапазоне $(130 \mathrm{GHz})$ в высоком магнитном поле и при низкой температуре $1.7 \mathrm{~K}$, т.е. в условиях высокого фактора Больцмана. Больцмановское распределение населенностей условно показано на рис. $5, b$ разным количеством шариков.

Спектр ЭПР комплекса $\mathrm{Mn}_{\mathrm{Ga}}^{2+}-\mathrm{O}_{\mathrm{As}}^{2-}$ в $\mathrm{GaAs}$ может быть описан спиновым гамильтонианом

$$
H=g \mu_{\mathrm{B}} \mathbf{B} \cdot \mathbf{S}+D\left[S_{z}^{2}-1 / 3 S(S+1)\right]+A \mathbf{I} \cdot \mathbf{S},
$$

где $B-$ магнитное поле, $g-$ изотропный $g$-фактор, равный $2.00, \mu_{\mathrm{B}}$ - магнетон Бора, $D$ - параметр тонкой структуры, $A-$ постоянная изотропного сверхтонкого взаимодействия, $S=5 / 2$ и $I=5 / 2-$ электронный и ядерный спины. 

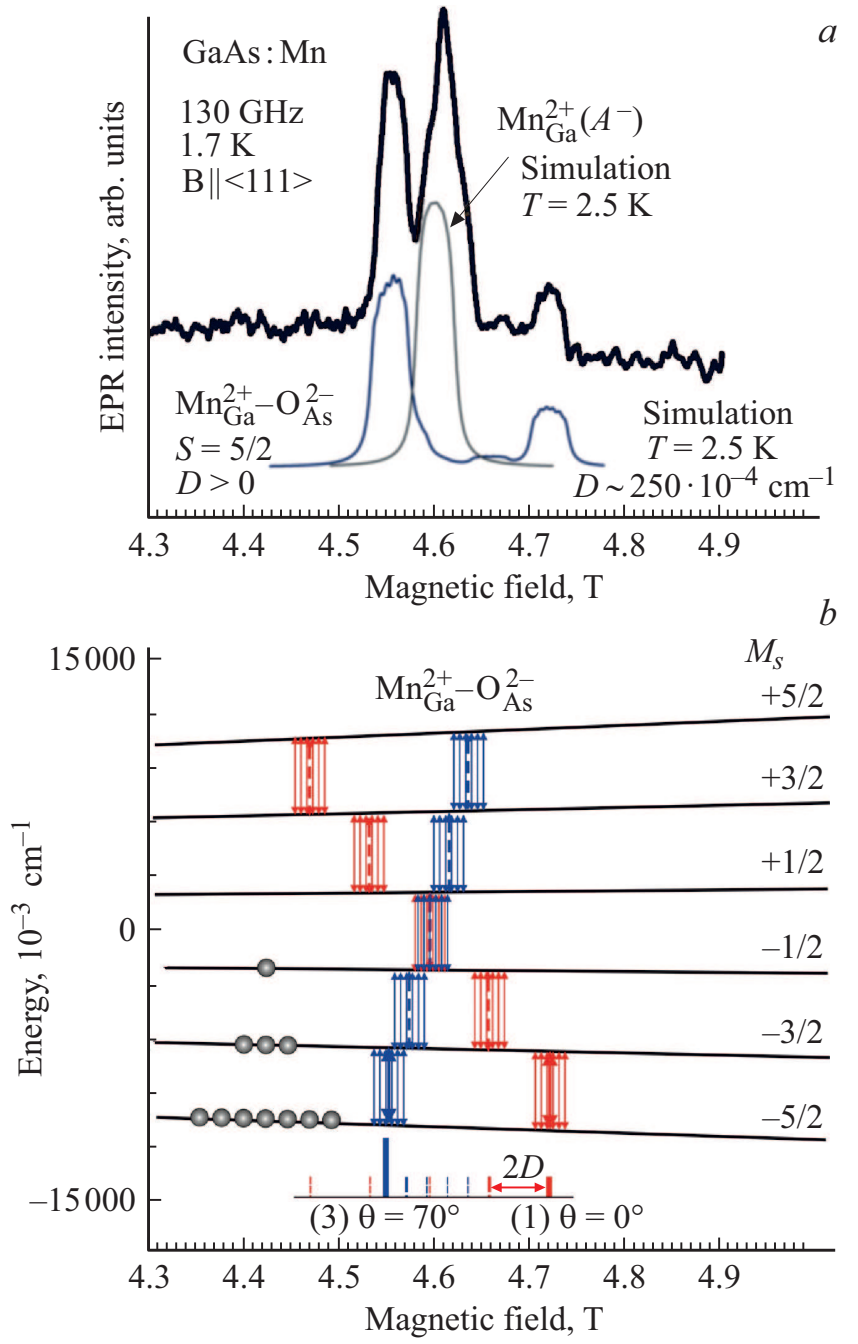

Рис. 5. (a) Спектр ЭПР комплексов марганца $\mathrm{Mn}_{\mathrm{Ga}}^{2+}-\mathrm{O}_{\mathrm{As}}^{2-}$ в кристалле GaAs: Mn, зарегистрированный в $2 \mathrm{~mm}$ диапазоне $(130 \mathrm{GHz})$ при низкой температуре $1.7 \mathrm{~K}$, т.е. в условиях высокого фактора Больцмана. (b) Схема уровней для четырех центров $\mathrm{Mn}_{\mathrm{Ga}}^{2+}-\mathrm{O}_{\mathrm{As}}^{2-}$, с магнитным полем ориентированным вдоль одного из эквивалентных направлений $\langle 111\rangle$ в кубическом кристалле GaAs (различие в уровнях для каждого из четырех центров находится в пределах ширины линии на графиках). В условиях высокого фактора Больцмана, показанного разным количеством шариков, будет наблюдаться эффективное поглощение микроволновой энергии только на переходах между нижними уровнями. $-5 / 2 \rightarrow-3 / 2$ с резким спадом интенсивности поглощения для остальных переходов. Этот эффект наблюдался в эксперименте и виден на симулированном спектре, представленном внизу $(a)$. Сильный сигнал в области $g=2.00$ принадлежит центрам $\mathrm{Mn}_{\mathrm{Ga}}^{2+}\left(A^{-}\right)$ в бездефектном окружении.

Рассчитанные энергетические уровни и ЭПР-переходы на частоте $130 \mathrm{GHz}$ для комплекса $\mathrm{Mn}_{\mathrm{Ga}}^{2+}-\mathrm{O}_{\mathrm{As}}^{2-}$ показаны на рис. $5, b$ соответствуют ориентации магнитного поля параллельно оси центра, совпадающих с однимой из четырех возможных ориентаций комплекса вдоль одной из молекулярных связей в тетра- эдре кристалла GaAs. Показаны шесть разрешенных переходов $\Delta M_{S}= \pm 1, \Delta m_{I}=0$ соответствуют ядерным переходам $m_{I}: 5 / 2 \rightarrow 5 / 2,3 / 2 \rightarrow 3 / 2,1 / 2 \rightarrow 1 / 2$, $-1 / 2 \rightarrow-1 / 2,-3 / 2 \rightarrow-3 / 2$, и $-5 / 2 \rightarrow-5 / 2$. Расчеты проводились с использованием программного пакета B. Грачева ViewEPR.

На рис. 5, $b$ показаны уровни энергии аксиального комплекса $\mathrm{Mn}_{\mathrm{Ga}}^{2+}-\mathrm{O}_{\mathrm{As}}^{2-}, S=5 / 2, I=5 / 2$ для магнитного поля, ориентированного параллельно одной из осей $\langle 111\rangle$. Ось симметрии одного из четырех возможных комплексов совпадает с направлением магнитного поля и в результате расщепление тонкой структуры является максимальным и равным 2D между соседними переходами. Эти переходы отмечены на рис. $5, b$ красным цветом. Для трех оставшихся комплексов ориентации оси центра составляют угол $110^{\circ}\left(70^{\circ}\right)$, их переходы совпадают и отмечены на рис. $5, b$ синим цветом. При вычислениях использовались следующие параметры спинового гамильтониана: $g=2.00, A=53 \cdot 10^{-4} \mathrm{~cm}^{-1}$, $D=250 \cdot 10^{-4} \mathrm{~cm}^{-1}$.

При термическом равновесии населенности уровней определяются распределением Больцмана, поэтому при понижении температуры преимущественно населены нижние уровни и ЭПР-переходы $-5 / 2 \leftrightarrow-3 / 2$ более интенсивны. Это приводит к асимметрии спектров ЭПР: при ориентации поля параллельно оси центра максимальную амплитуду должны иметь высокопольные пачки СТ-взаимодействия с центром тяжести, смещенным относительно $g=2.00$ на $4 D$ в высокие поля, а при перпендикулярной ориентации $\left(\theta=90^{\circ}\right)$ - пачки с центром, смешенным на $2 D$ в низкие поля. Для реализуемой в данном эксперименте ориентации для трех комплексов с углом $\theta=70^{\circ}$ крайняя линия смещена от центра на расстояние чуть меньшее $2 D$. На рис. $5, b$ более интенсивные линии показаны толстыми линиями, переходы меньшей интенсивности показаны штриховыми линиями (без учета СТ-0 структуры). На рис. 5, $a$ внизу показан спектр ЭПР, симулированный для температуры $1.7 \mathrm{~K}$. Видны два интенсивных сигнала, соответствующие переходам $M_{S}=-5 / 2 \rightarrow M_{S}=3 / 2$ для двух углов $\theta=0^{\circ}$ и $\theta=70^{\circ}$. Следует отметить, что отношение интенсивностей двух крайних компонент для сигналов $\theta=0^{\circ}$ на расстояниях $4 D$ и $2 D$ от центра дают информацию о температуре регистрации спектров ЭПР (в области температур ниже $10 \mathrm{~K}$ ).

На рис. 6 продемонстрировано влияние температуры на спектры ЭПР $\mathrm{Mn}_{\mathrm{Ga}}^{2+}-\mathrm{O}_{\mathrm{As}}^{2-}$ в $\mathrm{GaAs}$, обусловленное распределением Больцмана населенностей спиновых уровней в сильных магнитных полях. Спектры ЭПР комплексов $\mathrm{Mn}_{\mathrm{Ga}}^{2+}-\mathrm{O}_{\mathrm{As}}^{2-}$ и ионизированных акцепторов $\mathrm{Mn}_{\mathrm{Ga}}^{2+}$ симулированы при двух температурах, использовалась ширина линии $2 \mathrm{mT}$, в 2.5 раза меньше, чем при симуляции спектров на рис. $5, a$, чтобы проявить для наглядности разрешенную сверхтонкую структура марганца. Видно, что при повышении температуры увеличивается интенсивность перехода $-3 / 2 \rightarrow-1 / 2$ для ориентации $\theta=0^{\circ}$, начинает проявляться крайний переход $+3 / 2 \rightarrow+5 / 2$ 


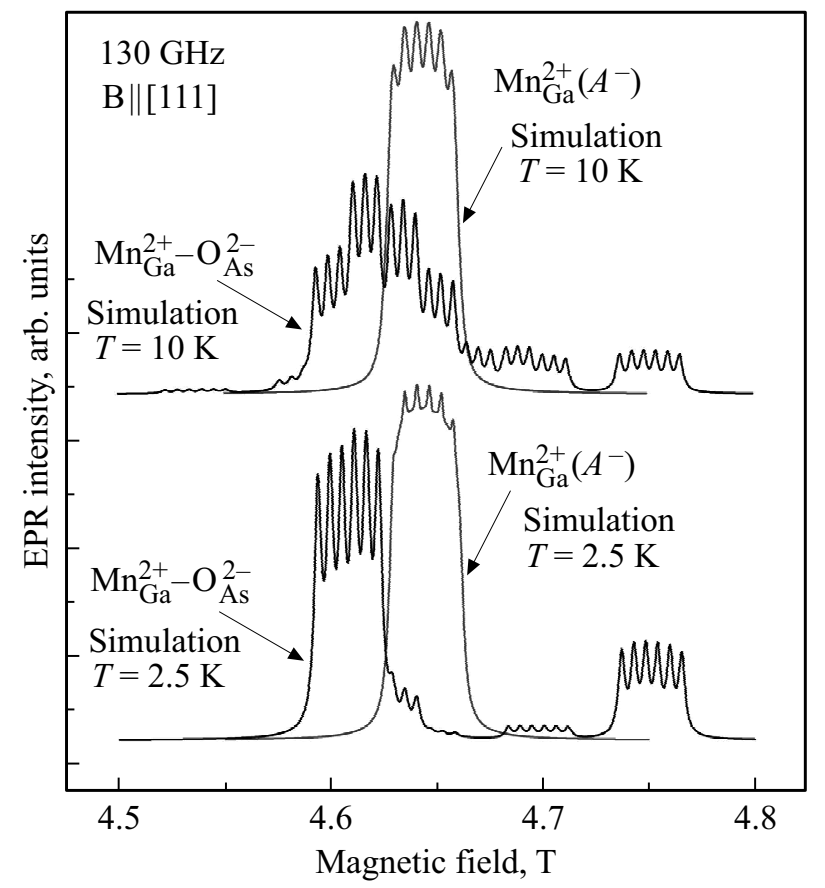

Рис. 6. Демонстрация влияния температуры на спектры ЭПР $\mathrm{Mn}_{\mathrm{Ga}}^{2+}-\mathrm{O}_{\mathrm{As}}^{2-}$ в $\mathrm{GaAs}$, обусловленного больцмановским распределением населенностей спиновых уровней в сильных магнитных полях. Спектры ЭПР комплексов $\mathrm{Mn}_{\mathrm{Ga}}^{2+}-\mathrm{O}_{\mathrm{As}}^{2-}$ и ионизированных акцепторов $\mathrm{Mn}_{\mathrm{Ga}}^{2+}$ симулированы для температуры $2.5 \mathrm{~K}$ и $10 \mathrm{~K}$. При симуляции использовалась ширина линии $2 \mathrm{mT}$, позволяющая проявить разрешенную сверхтонкую структура марганца.

(см. рис. 5,a), а также перераспределяются интенсивности других переходов для ориентации $\theta=70^{\circ}$. При дальнейшем повышении температуры будет наблюдаться спектр, симметричный относительно центрального перехода $-1 / 2 \rightarrow+1 / 2$. Сигнал ЭПР-центра $\mathrm{Mn}_{\mathrm{Ga}}^{2+}$ изменяется незначительно при повышении температуры, только улучшается разрешение сверхтонкой структуры. Тем не менее, это демонстрирует, что при низкой температуре разрешение СТ-структуры хуже, так как регистрируется только крайний переход $-5 / 2 \rightarrow-3 / 2$, в котором разброс параметров кристаллического поля проявляется сильнее, чем для центрального перехода $-1 / 2 \rightarrow+1 / 2$. Последний переход преобладает при повышении температуры.

\section{4. Заключение}

В работе проведены исследования ЭПР в высокочастотном диапазоне в сильных магнитных полях и низких температурах парамагнитных комплексов двух типов, представляющих собой один и тот же ионизированный акцептор $A^{-}$в виде $\mathrm{Mn}_{\mathrm{Ga}}^{2+}$ взаимодействующий с делокализованной дыркой с мелкими уровнями, комплекс $\mathrm{Mn}_{\mathrm{Ga}}^{2+} \mathrm{SH}$, и взаимодействующий с дыркой, локализованной на диамагнитном ионе примеси кислорода в со- седнем узле $\mathrm{As}$, комплекс $\mathrm{Mn}_{\mathrm{Ga}}^{2+}-\mathrm{O}_{\mathrm{As}}^{2-}$. Соответствующие модели показаны на рис. 2. Рассчитана система уровней в магнитном поле для двух комплексов, приведены переходы ЭПР и симулированы спектры ЭПР, наблюдаемые в условиях высокого фактора Больцмана, приводящего к заселению только нижних энергетических уровней. Показаны условия, при которых полная картина энергетических уровней может быть получена из результатов ЭПР-исследований. Подтверждены ранее предложенные модели с антиферромагнитным обменным взаимодействием в системе ионизированный акцептор марганца мелкая дырка, с нижним уровнем с $F=1$, а также установлен порядок уровней в комплексе ионизированный акцептор марганца - локализованная на кислороде дырка с $S=5 / 2$. Показано, что в исследуемых образцах нет магнитного упорядочения вплоть до высоких магнитных полей и температур $1.7 \mathrm{~K}$.

\section{Благодарности}

Авторы выражаются благодарность Н.С. Аверкиеву, В.Ф. Сапеге и К.Ф. Штельмаху за плодотворные дискуссии, стимулирующие проведение представленных в работе исследований, а также А.Д. Криворучко за участие в экспериментах.

\section{Финансирование работы}

Авторы выражают благодарность за финансовую поддержку Российскому научному фонду (проект № 20-12-00216).

\section{Конфликт интересов}

Авторы заявляют, что у них нет конфликта интересов.

\section{Список литературы}

[1] A.V. Komarov, S.M. Ryabchenko, O.V. Terletskii, I.I. Zheru, R.D. Ivanchuk. Zh. Eksp. Teor. Fiz. 73, 608 (1977). [Sov. Phys. JETP 46, 318 (1977)].

[2] J.A. Gaj, R.R. Galazka, M. Nawrocki. Solid State Commun. 25, 193 (1978).

[3] Semiconductor and Semimetals / Ed. J.K. Furdyna, J. Kossut. Academic, N.Y. (1988). V. 25.

[4] H. Ohno, A. Shen, F. Matsukura, A. Oiwa, A. Endo, S. Katsumoto, Y. Iye. Appl. Phys. Lett. 69, 363 (1996).

[5] T. Dietl, H. Ohno. Rev. Mod. Phys. 86, 187 (2014).

[6] J. Schneider, U. Kaufmann, W. Wilkening, M. Baeumler, F. Kohl. Phys. Rev. Lett. 59, 240 (1987).

[7] В.Ф. Мастеров, К.Ф. Штельмах, М.Н. Барбашов. ФТП 22, 654 (1988). [Sov. Phys. Semicond. 22, 4, 408 (1988)].

[8] Н.С. Аверкиев, А.А. Гуткин, О.Г. Красикова, Е.Б. Осипов, М.A. Рещиков. ФТП 23, 1, 73 (1989) [Sov. Phys. Semicond. 23, 44 (1989)].

[9] Н.С. Аверкиев, А.А. Гуткин. ФТТ 60, 2275 (2018) .

[10] M. Glunk, J. Daeubler, L. Dreher, S. Schwaiger, W. Schoch, R. Sauer, W. Limmer, A. Brandlmaier, S.T.B. Goennenwein, C. Bihler, M.S. Brandt. Phys. Rev. B 79, 195206 (2009). 
[11] D. Bimberg. Phys. Rev. B 18, 1794 (1978).

[12] V.F. Sapega, T. Ruf, M. Cardona. Phys. Status Solidi B 226, 339 (2001).

[13] H.C. Averkiev, A.A. Gutkin, E.B. Osipov, M.A. Reshchikov. Sov. Phys. Solid State 30, 438 (1988).

[14] M. Linnarsson, E. Janzén, B. Monemar, M. Kleverman, A. Thilderkvist. Phys. Rev. B 55, 6938 (1997).

[15] N.P. Baran, V.M. Maksimenko, Yu.G. Semenov, V.Ya. Bratus', A.V. Markov. JETP Lett. 55, 101 (1992).

[16] N. Almeleh, B. Goldstein. Phys. Rev. 12S, 1568 (1962).

[17] R. Bleekrode, J. Dieleman, H.J. Vegter. Phys. Lett. 2, 355 (1962).

[18] Y.J. Park, E.K. Kim, S.-K. Min, I.-W. Park, T.H. Yeom, H. Munekata, H. Kukimoto. J. Korean Phys. Society (Proc. Suppl.) 30, S113 (1997).

[19] M. Pawłowsky, M. Piersa, A. Wołoś, M. Palczewska, G. Strzelecka, A. Hruban, J. Gosk, M. Kamińska, A. Twardowski. Acta Phys. Polonica A 108, 825 (2005).

Редактор Д.В. Жуманов 